\title{
Monitoring of velocity changes based on seismic ambient noise: A brief review and perspective
}

\author{
Qing-Yu Wang ${ }^{1 *}$, and HuaJian Yao ${ }^{2,3,4}$ \\ 'University Grenoble Alpes, CNRS, ISTerre, Grenoble, France; \\ 2Laboratory of Seismology and Physics of Earth's Interior, School of Earth and Space Sciences, University of Science and Technology of China, Hefei 230026, \\ China; \\ ${ }^{3}$ Chinese Academy of Sciences Center for Excellence in Comparative Planetology, University of Science and Technology of China, Hefei 230026, China; \\ ${ }^{4}$ Mengcheng National Geophysical Observatory, University of Science and Technology of China, Mengcheng Anhui 233500, China \\ Key Points: \\ - We list some state-of-the-art monitoring methods \\ - We summarize substantial applications of noise-based monitoring at different scales and with different mechanisms \\ - The noise-based monitoring technique can be used to track changes in diverse physical parameters for both terrestrial and \\ extraterrestrial seismology
}

Citation: Wang, Q.-Y., and Yao, H. J. (2020). Monitoring of velocity changes based on seismic ambient noise: A brief review and perspective. Earth Planet. Phys., 4(5), 532-542. http://doi.org/10.26464/epp2020048

\begin{abstract}
Over the past two decades, the development of the ambient noise cross-correlation technology has spawned the exploration of underground structures. In addition, ambient noise-based monitoring has emerged because of the feasibility of reconstructing the continuous Green's functions. Investigating the physical properties of a subsurface medium by tracking changes in seismic wave velocity that do not depend on the occurrence of earthquakes or the continuity of artificial sources dramatically increases the possibility of researching the evolution of crustal deformation. In this article, we outline some state-of-the-art techniques for noise-based monitoring, including moving-window cross-spectral analysis, the stretching method, dynamic time wrapping, wavelet cross-spectrum analysis, and a combination of these measurement methods, with either a Bayesian least-squares inversion or the Bayesian Markov chain Monte Carlo method. We briefly state the principles underlying the different methods and their pros and cons. By elaborating on some typical noisebased monitoring applications, we show how this technique can be widely applied in different scenarios and adapted to multiples scales. We list classical applications, such as following earthquake-related co- and postseismic velocity changes, forecasting volcanic eruptions, and tracking external environmental forcing-generated transient changes. By monitoring cases having different targets at different scales, we point out the applicability of this technology for disaster prediction and early warning of small-scale reservoirs, landslides, and so forth. Finally, we conclude with some possible developments of noise-based monitoring at present and summarize some prospective research directions. To improve the temporal and spatial resolution of passive-source noise monitoring, we propose integrating different methods and seismic sources. Further interdisciplinary collaboration is indispensable for comprehensively interpreting the observed changes.
\end{abstract}

Keywords: ambient noise correlation; noise-based monitoring; seismic wave velocity changes; the evolution of physical properties of the crust

\section{Introduction}

Studying the stress state of the crust is essential to understand the mechanisms relevant to various tectonic and nontectonic processes of the earth. Monitoring seismic wave velocity changes is a useful tool for probing changes in the stress state of the crust, as has been verified both theoretically and experimentally (Birch, 1961; Nur and Simmons, 1969; O'Connell and Budiansky, 1974;

Correspondence to: Q.-Y. Wang, qingyu.wang@univ-grenoble-alpes.fr Received 11 MAY 2020; Accepted 02 JUN 2020.

Accepted article online 17 JUL 2020.

C) 2020 by Earth and Planetary Physics.
Schoenberg, 1980; Yamamura et al., 2003). The existence of persistent and extensive seismic noise allows us to reconstruct the Green's function by continuously cross- or auto-correlating seismic ambient noise recordings (Aki, 1957; Claerbout, 1968; Lobkis and Weaver, 2001; Campillo and Paul, 2003; Shapiro and Campillo, 2004; Larose et al., 2005a; Campillo, 2006, 2011). Thus, this noise allows us to monitor the seismic wave velocity changes with time. By measuring the changes in seismic velocity, we can investigate the stress state of the crust at depth and continuously in time, which provides essential constraints on dynamic processes in the crust, such as those attributable to earthquakes, volcanoes, and other activities. The ambient noise-based monitoring method can 
also be used to monitor subsurface velocity changes caused by various types of environmental changes. In addition, cross-correlation noise monitoring can be applied on small scales, such as at landslides and reservoirs, for possible disaster prediction and as an early warning system. Here we briefly review the methods of ambient seismic noise-based monitoring and the main fields where it can be applied. We then discuss some prospective research topics that are urgently needed and possible directions for future development.

\section{Methodology of Ambient Seismic Noise-Based Monitoring}

Conventional ambient noise-based seismic wave velocity monitoring is a coda wave-based technology collectively referred to as coda wave interferometry. The concept of coda wave-based seismic velocity monitoring was from Poupinet et al. (1984), who retrieved the subtle phase shift of waveforms between earthquake doublets along seismograms. Snieder et al. (2002) later proposed a similar concept to measure the time change by using different lapse time moving windows to evaluate the relative seismic wave velocity changes by linear regression. This evaluation assumes that changes in the wavefield are homogeneous; therefore, they obey a linear relationship in which a travel-time shift is opposite seismic velocity changes:

$$
\delta t / t=-\delta v / v
$$

Four different methods are mainly used for measuring the travel time shift based on coda waves. Brenguier et al. (2008a, b) and Clarke et al. (2011) applied what they termed a moving-window cross-spectral analysis to continuously record noise cross-correlations. A moving-window cross-spectral analysis is a frequency-domain analysis based on measuring the phase shift in a sliding window of specific lengths. Through two linear regressions, we can obtain the velocity changes between any two cross-correlation functions. This method requires us to define the width of the sliding window, the lapse time on the cross-correlation function, and a set of quality filtering restrictions, among others.

Alternatively, the stretching method (Lobkis and Weaver, 2003; Sens-Schönfelder and Wegler, 2006) is based on a time-domain analysis. It works by optimizing the cross-correlation coefficient between any two comparable cross-correlation functions after deforming one of them with a stretching coefficient. The changes in seismic velocity are equal to the stretching coefficient producing the maximum cross-correlation coefficient. This method can potentially be polluted by changes in the spectrum of noise sources (Zhan ZW et al., 2013).

Along with the two classical methods for measuring the time shift, there is dynamic time warping by Mikesell et al. (2015). Dynamic time warping utilizes all the information, the time shift from coda waves as well as the amplitude and decoherence information. Recently, Mao SJ et al. (2020) proposed a new approach based on a wavelet cross-spectrum analysis, a continuous wavelet transform. This approach provides optimal time-frequency joint resolution with full lapse time measurement. This method has a powerful computational advantage for multiple bands, and thus depth analysis. All four methods of measuring the time shift can further be used with linear inversion, based on the relationship below by Brenguier et al. (2014). Brenguier et al. (2014) showed that the difference in relative seismic velocity changes at time $j$ and time $i$ is approximately equal to the change between the two times:

$$
\delta v_{j}-\delta v_{i} \approx \delta v_{i j}
$$

where $i$ and $j$ indicate any two different times. This inversion-supplemented procedure helps avoid the choice of an arbitrary reference correlation function by the usual stacking. Accurate continuous velocity changes are retrieved by using a Bayesian leastsquares inversion. This inversion procedure improves the precision of the measurements by separately computing velocity changes for all the possible correlation functions for each station pair.

A regularization during the inversion allows us to focus on either rapid or long-term changes in seismic wave velocity. When dealing with high-temporal-resolution data or long time series, the inversion of the matrix will require a relatively large storage volume. Taylor and Hillers (2020) developed a new method of determining the seismic velocity time series by using a Bayesian Markov chain Monte Carlo (MCMC) approach. This method can effectively tackle the problem of having a large volume of data. The MCMC seeks to construct a full posterior probability distribution of the changes in seismic velocity. It provides a robust way to compute the time series of velocity changes by incorporating the information on measurement uncertainty.

The four measurement methods together with the further inversion and MCMC methods, which can increase measurement stability, are the most commonly used and updated techniques. They cover almost all the possibilities for conventional coda wavebased monitoring. It provides stable time series of seismic velocity changes at different depths. However, the disadvantage is that coda wave-based monitoring is often hindered by insufficient spatial resolution and difficulty in locating depth because of the complexity of the scattering paths of waves, and thus the sensitivity kernel of coda waves.

To avoid insufficient spatial resolution and the difficulty of determining the specific depth when using coda waves, Brenguier et al. (2020), Takano et al. (2020), and Mordret et al. (2020) began to do the monitoring using ballistic waves, including both body waves and surface waves (both the fundamental mode and the first overtone of Rayleigh waves) recovered from the seismic noise correlation. This technology relies on the deployment of smallscale targeted dense arrays, which have developed rapidly in recent years. These stations are usually arranged around critical faults; hence, the technology is essential for accurately tracking small-velocity changes within small areas adjacent to faults.

This new ballistic wave-based methodology provides better depth localization of the detected changes in seismic velocity. At the same time, it relies on the dense array to reduce possible bias from the high intrinsic sensitivity of reconstructed ballistic waves to the noise source properties. Currently, this is the main limitation encountered with ballistic wave-based monitoring. In contrast, coda wave-based monitoring is less sensitive to the noise source properties because of the phase velocity bias resulting 
from the anisotropic distribution of ambient noise energy (Yao $\mathrm{HJ}$ et al., 2009). One can successfully apply coda wave-based monitoring even without perfect reconstruction of the Green's function (Hadziioannou et al., 2009). Froment et al. (2010), Weaver et al. (2011), and Colombi et al. (2014) also estimated the travel time error by considering different distribution sources, and they confirmed the stability of using coda waves to do the monitoring. This is because the long duration and disorder of the scattering process can enhance the signal-to-noise ratio and reduce the dependency of the correlation function quality on the noise source properties (Larose et al., 2008).

We usually apply these methods in the continuous Green's functions reconstructed from long-term recorded background signals for passive source-based noise monitoring. Similarly, we can apply these methods to signals obtained from active sources. The Green's functions obtained from active sources, both earthquakeproduced and artificial (Reasenberg and Aki, 1974; Karageorgi et al., 1992; Ikuta et al., 2002; Yamamura et al., 2003; Niu FL et al., 2008; Yang W et al., 2018; Wang BS et al., 2020), have excellent advantages in the signal to noise ratio, and controllable repeatability in time for the latter one. Therefore, monitoring with active sources can improve the accuracy of tracking instantaneous coseismic changes. This may progress the possible underestimated momentary velocity drop by the earthquake that can hardly be measured by passive wave-based monitoring because of the compromise between the temporal resolution and the convergence time of stability. For this reason, it is also possible to use direct waves other than coda waves to tackle the problem of spatial localization. However, we need to consider the cost of artificial seismic sources and the relatively limited detection scale. The combination of both seismic sources with the methods above will significantly improve the accuracy of detection.

\section{Application and Observation of Ambient Seismic Noise-Based Monitoring}

Of the different methods mentioned, noise-based monitoring has been applied at different scales and with different research objectives to investigate the responses of the crust under the impacts of various mechanical processes in the past dozen years or more. Here we summarize the main origins of changes in seismic wave velocity as dynamic and static stress-strain-related earthquake co- and postseismic procedures, pressure buildup, and magma migration in the volcanic area, external forces from environmental perturbations, and the activities of natural and humanmade reservoirs. In this section, we list some representative investigations of noise-based monitoring with different forcing origins.

\subsection{Earthquake-Related Co- and Postseismic Velocity Changes}

Studies of the mechanical responses of the earth's crust to large earthquakes can provide us with unique insights into the processes of stress buildup and release at depth (Bürgmann and Dresen, 2008). At this stage, we mainly observe a rapid coseismic velocity reduction followed by a slow postseismic exponential recovery process (Field et al., 1998; Wegler and Sens-Schönfelder,
2007; Brenguier et al., 2008a, Xu ZJ and Song XD, 2009; Sawazaki et al., 2009; Chen JH et al., 2010; Cheng $X$ et al., 2010; Zhao PP et al., 2012; Froment et al., 2013; Acarel et al., 2014; Brenguier et al., 2014; Liu ZK et al., 2014; Hong TK et al., 2017; Liu ZK et al., 2018; Wang QY et al., 2019; Poli et al., 2020). The predominant mechanism underlying the observed earthquake-related velocity drop is referred to as shallow dynamic shaking (Sleep, 2015) and deep stress changes by the earthquake. The classical observation by Brenguier et al. (2008a) in the San Andreas fault zone (Figure 1) suggests that the observed seismic velocity changes $(0.08 \%)$ should come from both coseismic damage in the shallow layers and a deep coseismic stress change and postseismic stress relaxation within the fault zone. A similar phenomenon has been explained both experimentally and numerically by nonlinear elastic behavior, including both anomalous nonlinear fast dynamics and slow dynamics (Lyakhovsky et al., 1997, 2009; Johnson and Sutin, 2005; Sens-Schönfelder et al., 2019).

Here we present a recent study by Wang QY et al. (2019) related to the $2011 M_{w}$ 9.0 Tohoku-Oki earthquake off the coast of Japan. The authors identified depth (frequency)-dependent seismic velocity changes. A shallow seismic velocity decrease $(\sim 0.07 \%$ in the 1-7 s period band; Figure 2a) was observed to be related to a mechanical weakening of the crust by the dynamic stress (represented by a map of the peak ground velocity (PGV); Figure $2 \mathrm{~b}$ ) associated with seismic waves. Brenguier et al. (2014) also studied the sensitivity of stress perturbations to changes in the seismic wave velocity due to the Tohoku-Oki earthquake. Their results revealed that the seismic wave velocity changes in the central volcanic region of Japan were highly susceptible to coseismic dynamic stress perturbations. Distinct from the map of changes in this relatively short period, the map of velocity changes in the $8-30 \mathrm{~s}$ band ( 0.04\%; Figure $2 \mathrm{c}$ ) disclosed that the static strain caused by the earthquake at depth dominated the velocity decrease over a relatively long period. This was the first observation of static strain-related decreases in co- and postseismic velocity.

\subsection{Seismic Velocity Changes in Volcanic Areas}

Another crucial application is relatively small-scale observation in volcanic regions (Brenguier et al., 2016). One of the most classic and representative cases is the observation of systematic seismic velocity decrease $(\sim 0.05 \%)$ as a precursor to the eruption of volcanoes at Piton de la Fournaise (Brenguier at al., 2008b; Figure 3). Similar volcanic process-related observations (Mordret et al., 2010; Sens-Schönfelder et al., 2014; Donaldson et al., 2017; Hirose et al., 2017) indicated that velocity changes were strongly correlated with the dilatation or compression of the volcanic system. The heterogeneous spatial stress distribution can lead to opposite seismic velocity responses to the same volcanic activity. Liu ZQ et al. (2019) separated the observed velocity changes at the Kilauea volcano into two phases, which they explained by compression from upward migration of the magma and the injection of magma into the veins and fractures.

Recently, Takano et al. (2020) also applied ballistic wave-based monitoring to the Piton de la Fournaise volcano. They showed that the velocity changes as a result of strain complexity in response to the subtle pressurization of the shallow magma reser- 


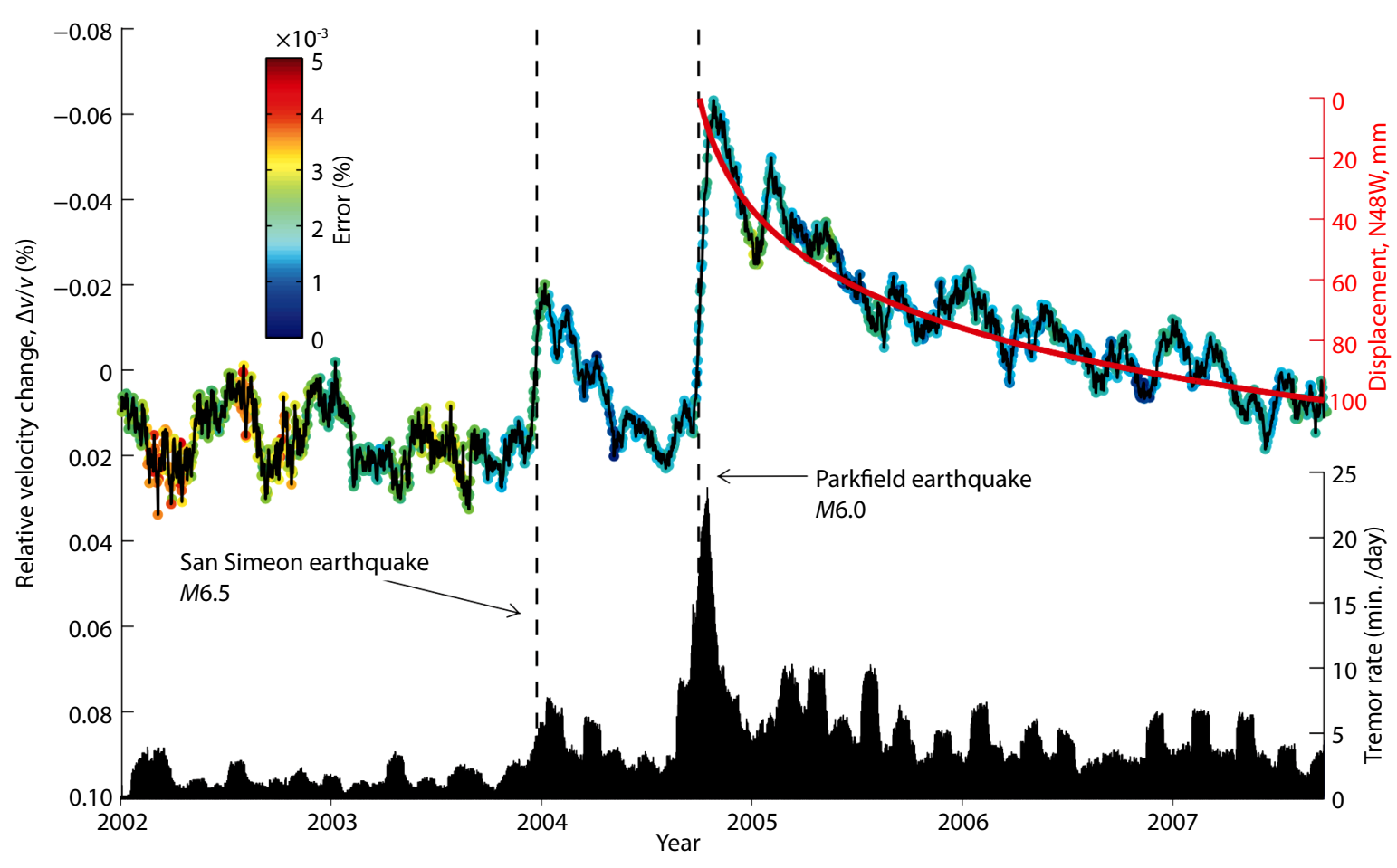

Figure 1. Seismic velocity changes, surface displacements from GPS, and tremor activity near Parkfield, California. The red curve represents the postseismic fault-parallel displacements along the San Andreas fault as measured by a GPS station. After Brenguier et al. (2008a).

voir. Noise-based monitoring thus provides a robust means of studying the processes of pressure buildup at depth and magma transport in the volcanic edifices so as to improve the ability to predict volcanic eruptions.

\subsection{Environmental Seismic Velocity Changes}

Apart from both tectonic- and volcanic-related seismic velocity changes, commonly existing environmental perturbations from multiple sources also play a significant role in the seismic wave velocity. Studying the transient velocity changes related to those environmental perturbations can help isolate the tectonic-related deformation and contribute to understanding the behavior of the crust under diverse external forcing mechanisms.

Groundwater- and rainfall-related seismic velocity decreases up to $\sim 10^{-2} \%$ to $10^{-1} \%$ that show the importance of hydraulic effects on seismic wave velocity have been widely discussed (SensSchönfelder and Wegler, 2006; Meier et al., 2010; Tsai, 2011; Hillers et al., 2014; Hotovec-Ellis et al., 2014; Wang QY at al., 2017; Lecocq et al., 2017; Poli et al., 2020). When rainfall increases, the infiltration of rainwater produces delayed pore pressure enhancements in the top kilometers of the crust. This in turn leads to a decrease in the shear modulus and consequently to decreases in the seismic wave velocity. Simultaneously, the velocity changes are delayed slightly because of hydraulic diffusion compared with the daily peaks in precipitation.

Figure 4b illustrates how rainfall in Kyushu, Japan, significantly decreased the velocity of the seismic waves in July 2011 and July 2012. Figure 4a shows a map of the averaged seismic velocity changes in July 2011 and July 2012 after Gaussian smoothing of a width of $100 \mathrm{~km}$. The highlighted velocity decreases beneath vol- canoes (Figure 4a, red triangles) also demonstrate the identical conclusion reached by Brenguier et al. (2014) of the high susceptibility of the volcanic area.

In the shallow layer, thermoelastic stress changes the velocity of seismic waves in an annual cycle (Meier et al., 2010; Richter et al., 2014; Hillers et al., 2015a; Lecocq et al., 2017), as does atmospheric pressure (Silver et al., 2007). Subtle tidal effects (Yamamura et al., 2003; Takano et al., 2014; Hillers et al., 2015b; Mao SJ et al., 2019; Wang BS et al., 2020) as well as permafrost freeze and melt (James et al., 2017) can also have a considerable effect in modulating the seismic wave velocity changes. In addition, snowfall, precipitation, and changes in sea surface height can cause direct elastic loading effects (Wang QY et al., 2017; Donaldson et al., 2019). These directly generated stresses usually have a compacting effect on the adjacent subsurface media. Media at different depths, distances, and fault orientations may have opposite responses to seismic velocity under the impact of the same environmental factor. Among them, the role of snowfall can be the most complicated. Not only does it generate direct elastic stress, but it also changes the pore pressure after melting when the temperature rises or when different geologic conditions are encountered (Mordret et al., 2016). The superposition and interaction of the environmental factors mentioned can generate combined effects on the underground media, which in turn affect the seismic wave velocity in a non-negligible way.

\subsection{Other Applications}

In addition to the major monitoring applications highlighted above, noise-based monitoring as it is currently being researched has further practical applications at different scales. The observed Rayleigh wave velocity drop (7\%) before a landslide caused by a 

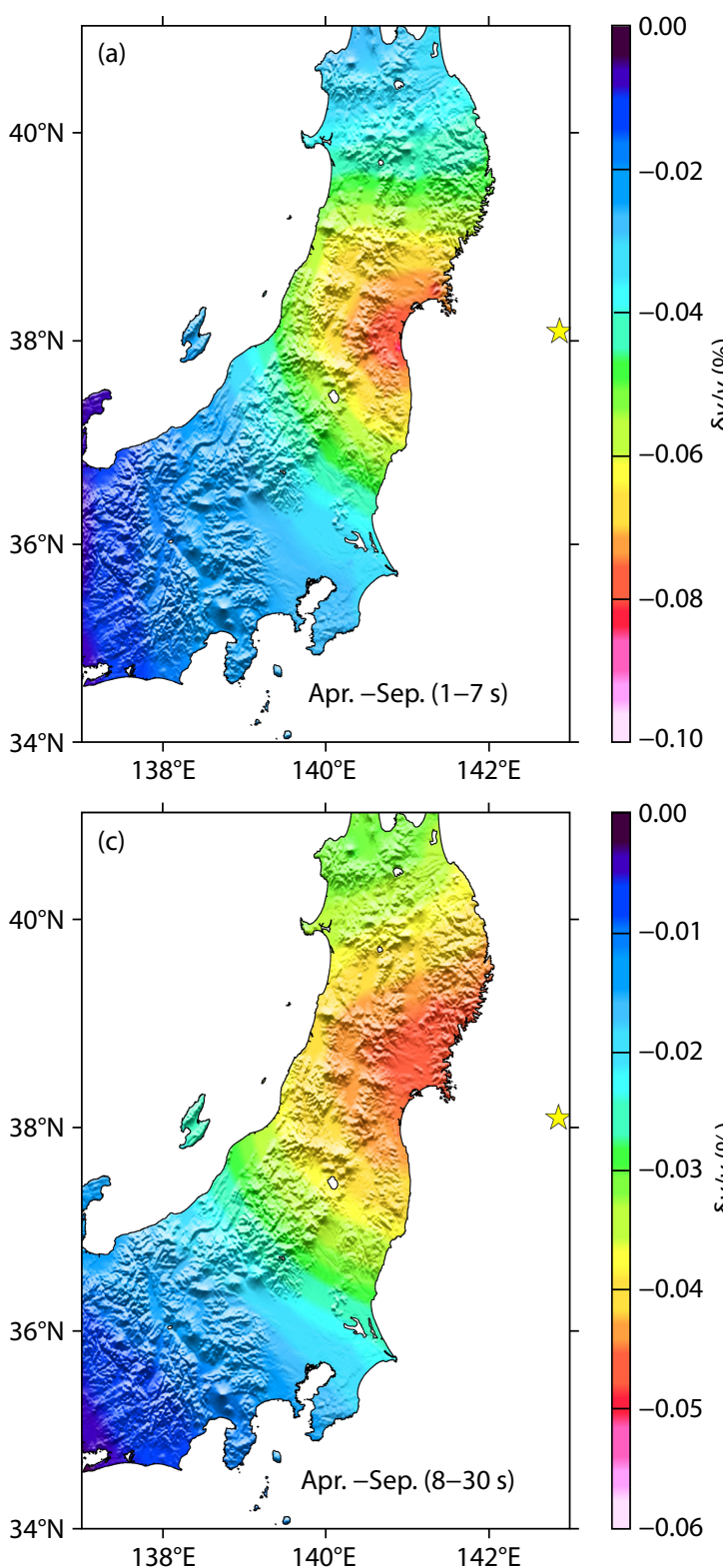
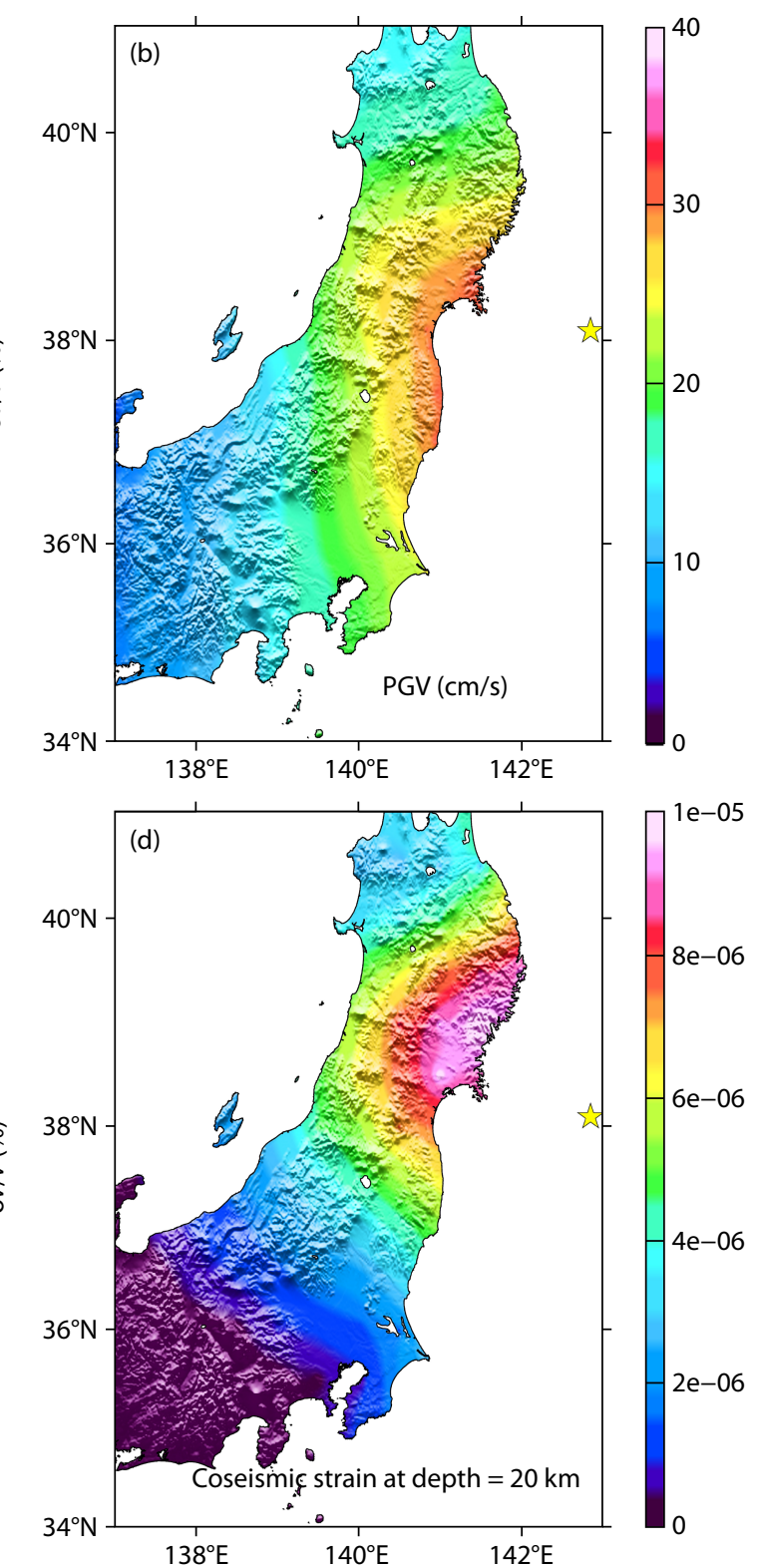

Figure 2. (a, c) Separate spatial seismic velocity changes measured at 1-7 s and 8-30 s. (b) Map of peak ground velocity (PGV, cm/s). (d) Coseismic strain modeled at a depth of $20 \mathrm{~km}$. After Wang et al. (2019).

decay in the mechanical rigidity of the clay suggests the possibility of applying ambient noise-based monitoring to predict failure on a local scale (Mainsant et al., 2012). Its failure prediction potential provides us with the opportunity to apply it in landslide monitoring and disaster prediction. Active source experiments both in the laboratory (Scuderi et al., 2016) and at a fault zone (Niu FL et al., 2008) have confirmed that the short-term velocity changes preceding failure can indicate earthquake nucleation. All these studies serve as essential references for tracking the changes in seismic wave velocity to predict and provide early warning of earthquakes and geological disasters. In addition, certain industrial activities, such as mine activities (Olivier et al., 2015) and fluid injection at geothermal sites (Hillers et al., 2015c; Obermann et al., 2015), can be tracked by the changes in velocity and decoherence in the waveform. Planès et al. (2016) and Olivier et al. (2017) successfully observed changes in the stress field of dams result- ing from changes in the groundwater level and in the porosity of the medium, which caused changes in the seismic wave velocity. Their work verifies the potential for tracking the internal deformation and pressure state at small-scale applications, such as at dams and reservoirs. Monitoring the evolution of the stress state at small-scale reservoirs is crucial for disaster prevention.

\section{Prospective Research in Ambient Seismic Noise- \\ Based Monitoring}

The preceding introduction to the methodology of ambient seismic noise-based monitoring and examples of its application verify the feasibility of this method for continuously tracking the evolution of the crustal medium to study the stress state of the earth's crust. Ambient seismic noise-based monitoring is a useful tool for observing the crustal responses to deformation sources of differ- 


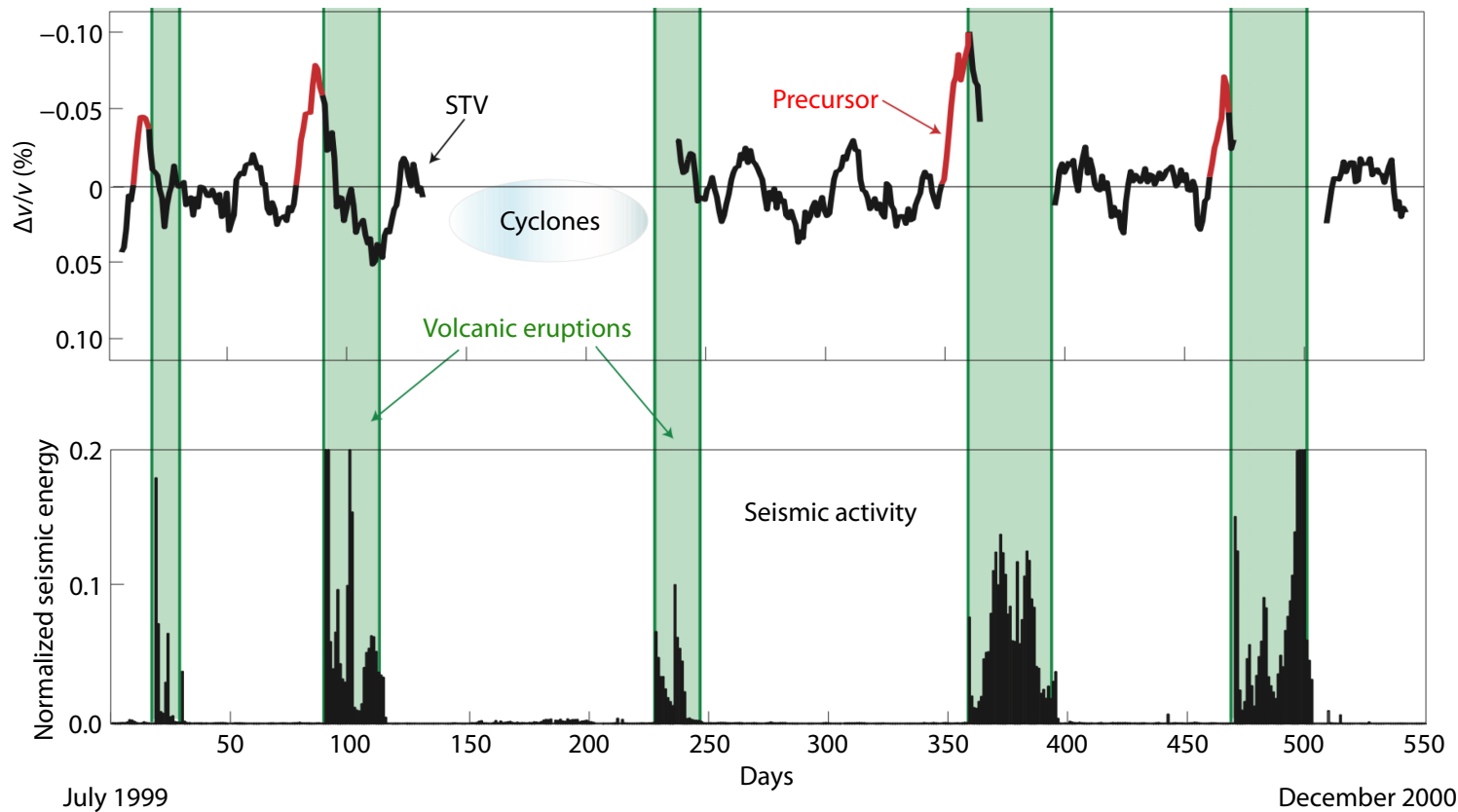

Figure 3. Seismic velocity reductions preceding eruptions of the Piton de la Fournaise volcano versus seismic energy, which is computed as the daily averaged root mean square (RMS) value of continuous seismic signals recorded at Piton de la Fournaise. The green columns show the periods of eruption by Brenguier et al. (2008b).

(a)

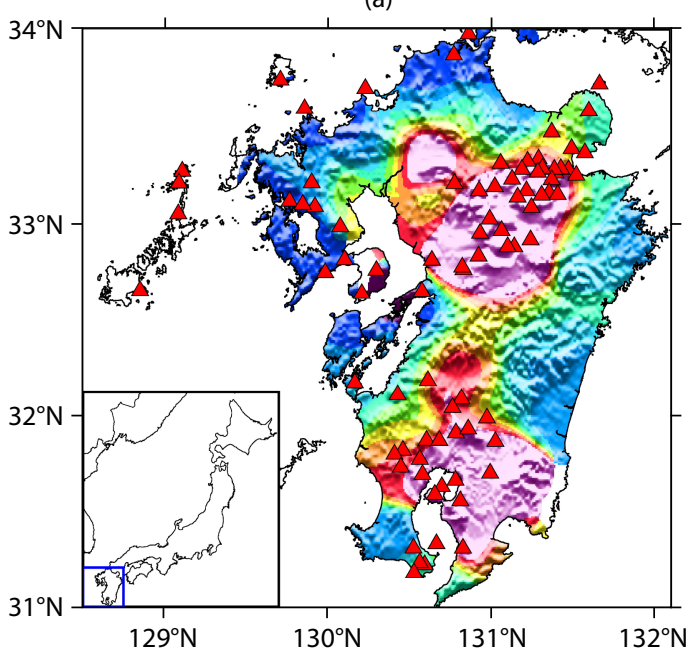

(b)

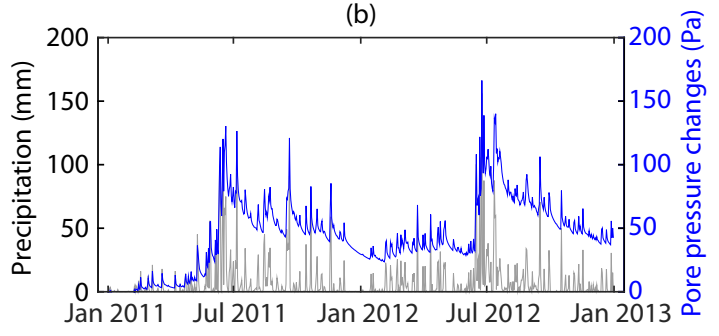

$0.00 \frac{a}{\frac{2}{2}}$

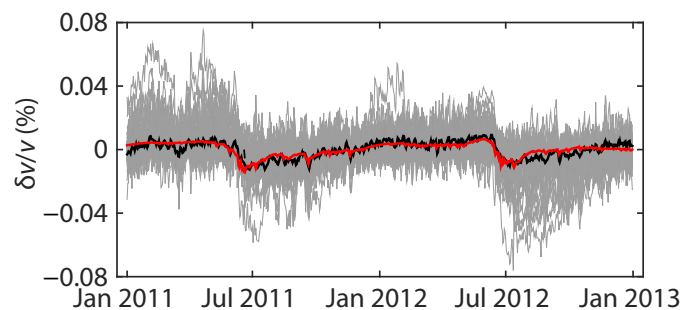

Figure 4. (a) Mapping of the mean seismic velocity changes in July 2011 and July 2012 at 74 High-Sensitivity Seismograph Network Japan (Hinet) stations. The red triangles represent the locations of quaternary volcanoes. (b) Upper record: daily mean precipitation for Kyushu (gray) and computed relative diffused pore pressure changes (blue) from 129 meteorological stations. Lower record: time series of seismic velocity changes for the Hi-net stations (gray) and the averaged $\delta v / v$ time series (black). The red curve represents the synthetic velocity changes. After Wang et al. (2017).

ent origins and is applicable from a small laboratory scale to local or regional scales. Further research is also needed in the following areas to improve noise-based monitoring.

Regarding the spatial resolution, current 2-D or 3-D imaging based on coda wave monitoring usually proceeds from a linear interpolation or inversion, and the analytical solution of the coda wave sensitivity kernel is based on the diffusion regime or radiative transfer theory. The sensitivity kernel is usually calculated un- der the assumption of isotropic scattering with a uniform energy velocity and transport mean free path (Pacheco and Snieder, 2005; Obermann et al., 2013a, b, 2014, 2019; Mayor et al., 2014; Planès et al., 2014; Margerin et al., 2016; Zhang YX et al., 2016; Nakahara and Emoto, 2017). Figure 5 illustrates a case by Obermann et al. (2019) showing that the linear combination of kernels from the body and surface waves is effective in constraining the depth of changes in a 3-D multiple-scattering medium. 

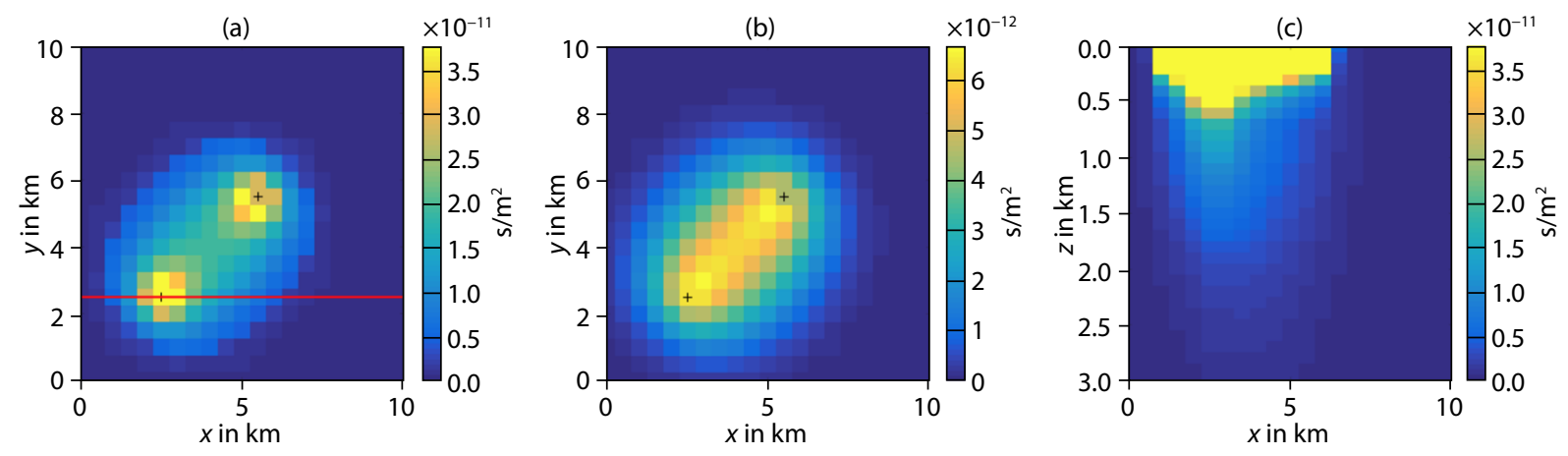

Figure 5. Combined coda kernel from 3-D body- and surface-wave kernels by Obermann et al. (2019). Horizontal slices at depths of $0.5 \mathrm{~km}$ (a) and $1.5 \mathrm{~km}$ (b) and a vertical slice (c) along the red line indicated in (a). The crosses mark the source and receiver positions.

We can see greater sensitivity around the two stations, and the shallow part seems more important than the deep part. Using similar sensitivity kernels, we can localize the measured travel time perturbations in the space and interpret the origins of the deformation more accurately. However, because of the complexity of the travel paths, we currently do not have an exact sensitivity kernel that can accurately characterize the travel time perturbations of coda waves and thus localize the measured changes with precision. Therefore, further studies of 2-D and 3-D scattered wave sensitivity kernels are imperative. With ballistic wave-based monitoring, spatial localization becomes relatively simple. However, the temporal resolution and the stability of the velocity changes are lower than those of coda waves. Knowing how to improve the signal-to-noise ratio of ballistic wave-based monitoring will thus become essential.

In terms of the temporal resolution, the current minimum detectable temporal resolution of passive-source noise monitoring is usually as little as one day or one hour according to the convergence rate of the Green's functions at different frequencies. With active sources, the repeatability of the Green's function is controllable and so is the temporal resolution. The ability to capture instantaneous changes can contribute to a better understanding of the momentary stress changes of earthquakes or other ruptures or volcanic eruptions, which will advance knowledge of their mechanisms and processes. Therefore, improving the temporal resolution will also become a vital research objective.

Pei SP et al. (2019) observed pronounced coseismic velocity reductions ( 4\%) after the 2008 Wenchuan earthquake by tracking the travel time changes in $\mathrm{Pg}$ waves of earthquakes more precisely. They located changes in depth ranging from 2 to $20 \mathrm{~km}$ with a 3-D tomographic inversion. This order of magnitude is much higher than the classical results from coda wave-based methods. Consequently, the existence of repetitive events from both artificial sources (Yang W et al., 2018; Wang QY et al., 2019) and repeating earthquakes (Sawazaki et al., 2015) extends the possibility of the combined use (Hirose et al., 2017) of ambient noise and other sources to detect changes in seismic wave velocity. Multiple approaches to monitoring can also effectively complement each other to improve the temporal and spatial resolution and extend the scope and sustainability of a region that can be monitored.
With the powerful computing capabilities currently available, realtime noise monitoring is becoming particularly realistic. Continuous changes in a seismic time series can be measured while being recorded in permanent seismic networks. It is necessary to analyze the local environmental factors simultaneously to distinguish among them and correct possible transient velocity changes related to nontectonic forcing. The purpose of real-time monitoring is to detect possible velocity anomalies in order to forecast potential disasters. Thus, statistical analysis of the seismic wave speed becomes vital follow-up work. Nakahara et al. (2020) used the extensive noise monitoring results in Japan to conduct a statistical analysis to quantify and detect velocity anomalies associated with volcanic eruptions or earthquakes. According to the mathematical distribution law, further criteria can be added to the real-time monitoring system automatically to predict possible anomalies.

With the acquisition of new, continuous seismic data and information from other planets (e.g., the moon and Mars), researchers have successfully applied the noise-based correlation technique to monitor seismic velocity changes on the moon (Larose et al., 2005b; Sens-Schönfelder and Larose, 2010). These applications investigate the moon by using diffusive waves in a frequency range outside of and higher than the typical microseismic bands. They have succeeded in extracting the Rayleigh waves by cross-correlating seismic noise recordings. The results have disclosed dynamic lunar processes related to the subsurface temperature, which Tanimoto et al. (2008) verified by comparing the amplitude of Rayleigh waves and the statistics on thermal moonquakes. Schimmel et al. (2018) verified the emergence of Rayleigh waves and normal modes by testing different correlational approaches with one station recording. The applications described above show the feasibility of applying the noise cross-correlation technique in extraterrestrial seismology for future planetary missions. The ambient noise correlation technology can be an effective method of investigating the internal structure of planets and following their temporal deformation.

In addition to monitoring changes in seismic wave velocity, the continuous Green's function provided by noise cross-correlation can track changes in other physical parameters of the crust over time. Obermann et al. (2014) located independent changes in seismic velocity and scattering properties of the crust from waveform decoherence to provide complementary information on the 
crustal evolution. Hirose et al. (2019) studied changes in the scattering and intrinsic absorption parameters of the crust based on envelopes of the cross-correlations. Durand et al. (2011) reported temporal variations in the polarization of surface waves that revealed changes in the orientation of distributed cracks in the medium. By studying multiple physical parameters independently, they can restrict each other and better explain the nature of the changes.

It is also worth noting that instead of seismic methods, other geophysical tools, such as geodesy, can also be applied to monitor the changes in underground physical properties. An interdisciplinary study has great potential to lead to a more comprehensive perspective on the underground stress field and its physical processes.

\section{Acknowledgments}

This work was supported by the Strategic Priority Research Program of the Chinese Academy of Sciences (grant no. XDB 41000000) and the China Seismic Experiment Site, China Earthquake Administration (project code 2018CSES0101). Qing-Yu Wang received funding from the European Research Council (ERC) under the European Union Horizon 2020 Research and Innovation Program (grant agreement 742335, F-IMAGE).

\section{References}

Acarel, D., Bulut, F., Bohnhoff, M., and Kartal, R. (2014). Coseismic velocity change associated with the 2011 Van earthquake (M 7.1): Crustal response to a major event. Geophys. Res. Lett., 41(13), 4519-4526. https://doi.org/10.1002/2014GL060624

Aki, K. (1957). Space and time spectra of stationary stochastic waves, with special reference to microtremors. Bull. Earthq. Res. Inst., 35(3), 415-456.

Birch, F. (1961). The velocity of compressional waves in rocks to 10 kilobars: 2. J. Geophys. Res., 66(7), 2199-2224. https://doi.org/10.1029/JZ066i007p02199

Brenguier, F., Campillo, M., Hadziioannou, C., Shapiro, N. M., Nadeau, R. M., and Larose, E. (2008a). Postseismic relaxation along the San Andreas fault at Parkfield from continuous seismological observations. Science, 321(5895), 1478-1481. https://doi.org/10.1126/science.1160943

Brenguier, F., Shapiro, N. M., Campillo, M., Ferrazzini, V., Duputel, Z., Coutant, O., and Nercessian, A. (2008b). Towards forecasting volcanic eruptions using seismic noise. Nat. Geosci., 1(2), 126-130. https://doi.org/10.1038/ngeo104

Brenguier, F., Campillo, M., Takeda, T., Aoki, Y., Shapiro, N. M., Briand, X., Emoto, K., and Miyake, H. (2014). Mapping pressurized volcanic fluids from induced crustal seismic velocity drops. Science, 345(6192), 80-82. https://doi.org/10.1126/science. 1254073

Brenguier, F., Rivet, D., Obermann, A., Nakata, N., Boué, P., Lecocq, T., Campillo, M., and Shapiro, N. (2016). 4-D noise-based seismology at volcanoes: Ongoing efforts and perspectives. J. Volcanol. Geoth. Res., 321, 182-195. https://doi.org/10.1016/j.jvolgeores.2016.04.036

Brenguier, F., Courbis, R., Mordret, A., Campman, X., Boué, P., Chmiel, M., Takano, T., Lecocq, T., Van der Veen, W., ... Hollis, D. (2020). Noise-based ballistic wave passive seismic monitoring. Part 1: Body waves. Geophys. J. Int., 221(1), 683-691. https://doi.org/10.1093/gji/ggz440

Bürgmann, R., and Dresen, G. (2008). Rheology of the lower crust and upper mantle: Evidence from rock mechanics, geodesy, and field observations. Annu. Rev. Earth Planet. Sci., 36, 531-567.

https://doi.org/10.1146/annurev.earth.36.031207.124326

Campillo, M., and Paul, A. (2003). Long-range correlations in the diffuse seismic coda. Science, 299(5606), 547-549. https://doi.org/10.1126/science.1078551

Campillo, M. (2006). Phase and correlation in 'random' seismic fields and the reconstruction of the Green function. Pure Appl. Geophys., 163(2), 475-502. https://doi.org/10.1007/s00024-005-0032-8

Campillo, M., Sato, H., Shapiro, N. M., and van der Hilst, R. D. (2011). New developments on imaging and monitoring with seismic noise. C. R. Geosci., 343(8-9), 487-495. https://doi.org/10.1016/j.crte.2011.07.007

Chen, J. H., Froment, B., Liu, Q. Y., and Campillo, M. (2010). Distribution of seismic wave speed changes associated with the 12 May $2008 \mathrm{Mw} 7.9$ Wenchuan earthquake. Geophys. Res. Lett., 37(18), L18302. https://doi.org/10.1029/2010GL044582

Cheng, X., Niu, F. L., and Wang, B. S. (2010). Coseismic velocity change in the rupture zone of the $2008 \mathrm{Mw} 7.9$ Wenchuan earthquake observed from ambient seismic noise. Bull. Seismol. Soc. Am., 100(5B), 2539-2550. https://doi.org/10.1785/0120090329

Claerbout, J. F. (1968). Synthesis of a layered medium from its acoustic transmission response. Geophysics, 33(2), 264-269. https://doi.org/10.1190/1.1439927

Clarke, D., Zaccarelli, L., Shapiro, N. M., and Brenguier, F. (2011). Assessment of resolution and accuracy of the Moving Window Cross Spectral technique for monitoring crustal temporal variations using ambient seismic noise. Geophys. J. Int., 186(2), 867-882. https://doi.org/10.1111/j.1365246X.2011.05074.x

Colombi, A., Chaput, J., Brenguier, F., Hillers, G., Roux, P., and Campillo, M. (2014). On the temporal stability of the coda of ambient noise correlations. C. R. Geosci., 346(11-12), 307-316. https://doi.org/10.1016/j.crte.2014.10.002

Donaldson, C., Caudron, C., Green, R. G., Thelen, W. A., and White, R. S. (2017). Relative seismic velocity variations correlate with deformation at Kīlauea volcano. Sci. Adv., 3(6), e1700219. https://doi.org/10.1126/sciadv.1700219

Donaldson, C., Winder, T., Caudron, C., and White, R. S. (2019). Crustal seismic velocity responds to a magmatic intrusion and seasonal loading in Iceland's Northern Volcanic Zone. Sci. Adv., 5(11), eaax6642. https://doi.org/10.1126/sciadv.aax6642

Durand, S., Montagner, J. P., Roux, P., Brenguier, F., Nadeau, R. M., and Ricard, Y. (2011). Passive monitoring of anisotropy change associated with the Parkfield 2004 earthquake. Geophys. Res. Lett., 38(13), L13303. https://doi.org/10.1029/2011GL047875

Field, E. H., Zeng, Y. H., Johnson, P. A., and Beresnev, I. A. (1998). Nonlinear sediment response during the 1994 Northridge earthquake: Observations and finite source simulations. J. Geophys. Res.: Solid Earth, 103(B11), 26869-26883. https://doi.org/10.1029/98jb02235

Froment, B., Campillo, M., Roux, P., Gouédard, P., Verdel, A., and Weaver, R. L. (2010). Estimation of the effect of nonisotropically distributed energy on the apparent arrival time in correlations. Geophysics, 75(5), SA85-SA93. https://doi.org/10.1190/1.3483102

Froment, B., Campillo, M., Chen, J. H., and Liu, Q. Y. (2013). Deformation at depth associated with the 12 May $2008 \mathrm{Mw} 7.9$ Wenchuan earthquake from seismic ambient noise monitoring. Geophys. Res. Lett., 40(1), 78-82. https://doi.org/10.1029/2012GL053995

Hadziioannou, C., Larose, E., Coutant, O., Roux, P., and Campillo, M. (2009). Stability of monitoring weak changes in multiply scattering media with ambient noise correlation: Laboratory experiments. J. Acoust. Soc. Am., 125(6), 3688-3695. https://doi.org/10.1121/1.3125345

Hillers, G., Campillo, M., and Ma, K. F. (2014). Seismic velocity variations at TCDP are controlled by MJO driven precipitation pattern and high fluid discharge properties. Earth Planet. Sci. Lett., 391, 121-127. https://doi.org/10.1016/j.epsl.2014.01.040

Hillers, G., Ben-Zion, Y., Campillo, M., and Zigone, D. (2015a). Seasonal variations of seismic velocities in the San Jacinto fault area observed with ambient seismic noise. Geophys. J. Int., 202(2), 920-932. https://doi.org/10.1093/gji/ggv151

Hillers, G., Retailleau, L., Campillo, M., Inbal, A., Ampuero, J. P., and Nishimura, T. (2015b). In situ observations of velocity changes in response to tidal deformation from analysis of the high-frequency ambient wavefield. J. Geophys. Res.: Solid Earth, 120(1), 210-225. https://doi.org/10.1002/2014JB011318

Hillers, G., Husen, S., Obermann, A., Planès, T., Larose, E., and Campillo, M. (2015c). Noise-based monitoring and imaging of aseismic transient deformation induced by the 2006 Basel reservoir stimulation. Geophysics, 
80(4), KS51-KS68. https://doi.org/10.1190/geo2014-0455.1

Hirose, T., Nakahara, H., and Nishimura, T. (2017). Combined use of repeated active shots and ambient noise to detect temporal changes in seismic velocity: Application to Sakurajima volcano, Japan. Earth, Planets and Space, 69, 42. https://doi.org/10.1186/s40623-017-0613-7

Hirose, T., Nakahara, H., and Nishimura, T. (2019). A passive estimation method of scattering and intrinsic absorption parameters from envelopes of seismic ambient noise cross-correlation functions. Geophys. Res. Lett., 46(7), 3634-3642. https://doi.org/10.1029/2018GL080553

Hong, T. K., Lee, J., Chi, D., and Park, S. (2017). Seismic velocity changes in the backarc continental crust after the $2011 M_{\mathrm{w}} 9.0$ Tohoku-Oki megathrust earthquake. Geophys. Res. Lett., 44(21), 10997-11003. https://doi.org/10.1002/2017GL075447

Hotovec-Ellis, A. J., Gomberg, J., Vidale, J. E., and Creager, K. C. (2014). A continuous record of intereruption velocity change at Mount St. Helens from coda wave interferometry. J. Geophys. Res.: Solid Earth, 119(3), 2199-2214. https://doi.org/10.1002/2013JB010742

Ikuta, R., Yamaoka, K., Miyakawa, K., Kunitomo, T., and Kumazawa, M. (2002). Continuous monitoring of propagation velocity of seismic wave using ACROSS. Geophys. Res. Lett., 29(13), 5-1. https://doi.org/10.1029/2001GL013974

James, S. R., Knox, H. A., Abbott, R. E., and Screaton, E. J. (2017). Improved moving window cross-spectral analysis for resolving large temporal seismic velocity changes in permafrost. Geophys. Res. Lett., 44(9), 4018-4026. https://doi.org/10.1002/2016GL072468

Johnson, P., and Sutin, A. (2005). Slow dynamics and anomalous nonlinear fast dynamics in diverse solids. J. Acoust. Soc. Am., 117(1), 124-130. https://doi.org/10.1121/1.1823351

Karageorgi, E., Clymer, R., and McEvilly, T. V. (1992). Seismological studies at Parkfield. II. Search for temporal variations in wave propagation using vibroseis. Bull. Seismol. Soc. Am., 82(3), 1388-1415.

Larose, E., Derode, A., Clorennec, D., Margerin, L., and Campillo, M. (2005a). Passive retrieval of Rayleigh waves in disordered elastic media. Phys. Rev. E, 72(4), 046607. https://doi.org/10.1103/PhysRevE.72.046607

Larose, E., Khan, A., Nakamura, Y., and Campillo, M. (2005b). Lunar subsurface investigated from correlation of seismic noise. Geophys. Res. Lett., 32(16), L16201. https://doi.org/10.1029/2005GL023518

Larose, E., Roux, P., Campillo, M., and Derode, A. (2008). Fluctuations of correlations and Green's function reconstruction: Role of scattering. J. Appl. Phys., 103(11), 114907. https://doi.org/10.1063/1.2939267

Lecocq, T., Longuevergne, L., Pedersen, H. A., Brenguier, F., and Stammler, K. (2017). Monitoring ground water storage at mesoscale using seismic noise: 30 years of continuous observation and thermo-elastic and hydrological modeling. Sci. Rep., 7, 14241. https://doi.org/10.1038/s41598-017-14468-9

Liu, Z. K., Huang, J. L., Peng, Z. G., and Su, J. R. (2014). Seismic velocity changes in the epicentral region of the 2008 Wenchuan earthquake measured from three-component ambient noise correlation techniques. Geophys. Res. Lett., 41(1), 37-42. https://doi.org/10.1002/2013GL058682

Liu, Z. K., Huang, J. L., He, P., and Qi, J. J. (2018). Ambient noise monitoring of seismic velocity around the Longmenshan fault zone from 10 years of continuous observation. J. Geophys. Res.: Solid Earth, 123(10), 8979-8994. https://doi.org/10.1029/2018JB015986

Liu, Z. Q., Liang, C. T., Zhu, Z. J., Wang, L., Jiang, N. B., Wang, C. L., and Wu, Z. B. (2019). The complex velocity variation induced by the precipitation and the 2018 eruption of the Kilauea Volcano in Hawaii revealed by ambient noise. Seismol. Res. Lett., 90(6), 2154-2164. https://doi.org/10.1785/0220190053

Lobkis, O. I., and Weaver, R. L. (2001). On the emergence of the Green's function in the correlations of a diffuse field. J. Acoust. Soc. Am., 110(6), 3011-3017. https://doi.org/10.1121/1.1417528

Lobkis, O. I., and Weaver, R. L. (2003). Coda-wave interferometry in finite solids: Recovery of $\mathrm{P}$ - to $\mathrm{S}$ conversion rates in an elastodynamic billiard. Physical Review Letters, 90(25(Pt 1)), 254302. https://doi.org/10.1103/PhysRevLett.90.254302

Lyakhovsky, V., Reches, Z., Weinberger, R., and Scott, T. E. (1997). Non-linear elastic behaviour of damaged rocks. Geophys. J. Int., 130(1), 157-166.
https://doi.org/10.1111/j.1365-246X.1997.tb00995.x

Lyakhovsky, V., Hamiel, Y., Ampuero, J. P., and Ben-Zion, Y. (2009). Non-linear damage rheology and wave resonance in rocks. Geophys. J. Int., 178(2), 910-920. https://doi.org/10.1111/j.1365-246X.2009.04205.x

Mainsant, G., Larose, E., Brönnimann, C., Jongmans, D., Michoud, C., and Jaboyedoff, M. (2012). Ambient seismic noise monitoring of a clay landslide: Toward failure prediction. J. Geophys. Res.: Earth Surf., 117(F1), F01030. https://doi.org/10.1029/2011JF002159

Mao, S. J., Campillo, M., van der Hilst, R. D., Brenguier, F., Stehly, L., and Hillers, G. (2019a). High temporal resolution monitoring of small variations in crustal strain by dense seismic arrays. Geophys. Res. Lett., 46(1), 128-137. https://doi.org/10.1029/2018GL079944

Mao, S. J., Mordret, A., Campillo, M., Fang, H. J., and van der Hilst, R. D. (2020). On the measurement of seismic traveltime changes in the time-frequency domain with wavelet cross-spectrum analysis. Geophys. J. Int., 221(1), 550-568. https://doi.org/10.1093/gji/ggz495

Margerin, L., Planès, T., Mayor, J., and Calvet, M. (2016). Sensitivity kernels for coda-wave interferometry and scattering tomography: Theory and numerical evaluation in two-dimensional anisotropically scattering media. Geophys. J. Int., 204(1), 650-666. https://doi.org/10.1093/gji/ggv470

Mayor, J., Margerin, L., and Calvet, M. (2014). Sensitivity of coda waves to spatial variations of absorption and scattering: Radiative transfer theory and 2-D examples. Geophys. J. Int., 197(2), 1117-1137. https://doi.org/10.1093/gji/ggu046

Meier, U., Shapiro, N. M., and Brenguier, F. (2010). Detecting seasonal variations in seismic velocities within Los Angeles basin from correlations of ambient seismic noise. Geophys. J. Int., 181(2), 985-996. https://doi.org/10.1111/j.1365-246X.2010.04550.x

Mikesell, T. D., Malcolm, A. E., Yang, D., and Haney, M. M. (2015). A comparison of methods to estimate seismic phase delays: Numerical examples for coda wave interferometry. Geophys. J. Int., 202(1), 347-360. https://doi.org/10.1093/gji/ggv138

Mordret, A., Jolly, A. D., Duputel, Z., and Fournier, N. (2010). Monitoring of phreatic eruptions using interferometry on retrieved cross-correlation function from ambient seismic noise: Results from Mt. Ruapehu, New Zealand. J. Volcan. Geoth. Res., 191(1-2), 46-59. https://doi.org/10.1016/j.jvolgeores.2010.01.010

Mordret, A., Mikesell, T. D., Harig, C., Lipovsky, B. P., and Prieto, G. A. (2016). Monitoring southwest Greenland's ice sheet melt with ambient seismic noise. Sci. Adv., 2(5), e1501538. https://doi.org/10.1126/sciadv.1501538

Mordret, A., Courbis, R., Brenguier, F., Chmiel, M., Garambois, S., Mao, S. J., Boué, P., Campman, X., Lecocq, T., ... Hollis, D. (2020). Noise-based ballistic wave passive seismic monitoring-Part 2: Surface waves. Geophys. J. Int., 221(1), 692-705. https://doi.org/10.1093/gji/ggaa016

Nakahara, H., and Emoto, K. (2017). Deriving sensitivity kernels of coda-wave travel times to velocity changes based on the three-dimensional single isotropic scattering model. Pure Appl. Geophys., 174(1), 327-337. https://doi.org/10.1007/s00024-016-1358-0

Nakahara, H., Wang, Q., Hobiger, M., and Hirose, T. (2020). Statistical characteristics of seismic velocity changes measured by seismic interferometry. (In review)

Niu, F. L., Silver, P. G., Daley, T. M., Cheng, X., and Majer, E. L. (2008). Preseismic velocity changes observed from active source monitoring at the Parkfield SAFOD drill site. Nature, 454(7201), 204-208. https://doi.org/10.1038/nature07111

Nur, A., and Simmons, G. (1969). The effect of saturation on velocity in low porosity rocks. Earth Planet. Sci. Lett., 7(2), 183-193. https://doi.org/10.1016/0012-821X(69)90035-1

Obermann, A., Planès, T., Larose, E., and Campillo, M. (2013a). Imaging preeruptive and coeruptive structural and mechanical changes of a volcano with ambient seismic noise. J. Geophys. Res.: Solid Earth, 118(12), 6285-6294. https://doi.org/10.1002/2013JB010399

Obermann, A., Planès, T., Larose, E., Sens-Schönfelder, C., and Campillo, M. (2013b). Depth sensitivity of seismic coda waves to velocity perturbations in an elastic heterogeneous medium. Geophys. J. Int., 194(1), 372-382. 
https://doi.org/10.1093/gji/ggt043

Obermann, A., Froment, B., Campillo, M., Larose, E., Planès, T., Valette, B., Chen, J. H., and Liu, Q. Y. (2014). Seismic noise correlations to image structural and mechanical changes associated with the $M_{w} 7.92008$ Wenchuan earthquake. J. Geophys. Res.: Solid Earth, 119(4), 3155-3168. https://doi.org/10.1002/2013JB010932

Obermann, A., Kraft, T., Larose, E., and Wiemer, S. (2015). Potential of ambient seismic noise techniques to monitor the St. Gallen geothermal site (Switzerland). J. Geophys. Res.: Solid Earth, 120(6), 4301-4316. https://doi.org/10.1002/2014JB011817

Obermann, A., Planès, T., Larose, E., and Campillo, M. (2019). 4-D imaging of subsurface changes with coda waves: numerical studies of 3-D combined sensitivity kernels and applications to the $M_{\mathrm{w}} 7.9,2008$ Wenchuan earthquake. Pure Appl. Geophys., 176(3), 1243-1254. https://doi.org/10.1007/s00024-018-2014-7

O'Connell, R. J., and Budiansky, B. (1974). Seismic velocities in dry and saturated cracked solids. J. Geophys. Res., 79(35), 5412-5426. https://doi.org/10.1029/JB079i035p05412

Olivier, G., Brenguier, F., Campillo, M., Roux, P., Shapiro, N. M., and Lynch, R. (2015). Investigation of coseismic and postseismic processes using in situ measurements of seismic velocity variations in an underground mine. Geophys. Res. Lett., 42(21), 9261-9269. https://doi.org/10.1002/2015GL065975

Olivier, G., Brenguier, F., De Wit, T., and Lynch, R. (2017). Monitoring the stability of tailings dam walls with ambient seismic noise. Leading Edge, 36(4), 350a1-350a6. https://doi.org/10.1190/tle36040350a1.1

Pacheco, C., and Snieder, R. (2005). Time-lapse travel time change of multiply scattered acoustic waves. J. Acoust. Soc. Am., 118(3), 1300-1310. https://doi.org/10.1121/1.2000827

Pei, S. P., Niu, F. L., Ben-Zion, Y., Sun, Q., Liu, Y. B., Xue, X. T., Su, J. R., and Shao, Z. G. (2019). Seismic velocity reduction and accelerated recovery due to earthquakes on the Longmenshan fault. Nat. Geosci., 12(5), 387-392. https://doi.org/10.1038/s41561-019-0347-1

Planès, T., Larose, E., Margerin, L., Rossetto, V., and Sens-Schönfelder, C. (2014). Decorrelation and phase-shift of coda waves induced by local changes: Multiple scattering approach and numerical validation. Waves Random Complex Media, 24(2), 99-125. https://doi.org/10.1080/17455030.2014.880821

Planès, T., Mooney, M. A., Rittgers, J. B. R., Parekh, M. L., Behm, M., and Snieder, R. (2016). Time-lapse monitoring of internal erosion in earthen dams and levees using ambient seismic noise. Géotechnique, 66(4), 301-312. https://doi.org/10.1680/jgeot.14.P.268

Poli, P., Marguin, V., Wang, Q. Y., D'agostino, N., and Johnson, P. (2020). Seasonal and co-seismic velocity variation in the region of L'Aquila from single station measurements and implications for crustal rheology. J. Geophys. Res.: Solid Earth, 125, e2019JB019316. https://doi.org/10.1029/2019JB019316

Poupinet, G., Ellsworth, W. L., and Frechet, J. (1984). Monitoring velocity variations in the crust using earthquake doublets: An application to the Calaveras Fault, California. J. Geophys. Res.: Solid Earth, 89(B7), 5719-5731. https://doi.org/10.1029/JB089iB07p05719

Reasenberg, P., and Aki, K. (1974). A precise, continuous measurement of seismic velocity for monitoring in situ stress. J. Geophys. Res., 79(2), 399-406. https://doi.org/10.1029/JB079i002p00399

Richter, T., Sens-Schönfelder, C., Kind, R., and Asch, G. (2014). Comprehensive observation and modeling of earthquake and temperature-related seismic velocity changes in northern Chile with passive image interferometry. J. Geophys. Res.: Solid Earth, 119(6), 4747-4765. https://doi.org/10.1002/2013JB010695

Sawazaki, K., Sato, H., Nakahara, H., and Nishimura, T. (2009). Time-lapse changes of seismic velocity in the shallow ground caused by strong ground motion shock of the 2000 Western-Tottori earthquake, Japan, as revealed from coda deconvolution analysis. Bull. Seismol. Soc. Am., 99(1), 352-366. https://doi.org/10.1785/0120080058

Sawazaki, K., Kimura, H., Shiomi, K., Uchida, N., Takagi, R., and Snieder, R. (2015). Depth-dependence of seismic velocity change associated with the 2011 Tohoku earthquake, Japan, revealed from repeating earthquake analysis and finite-difference wave propagation simulation. Geophys. J. Int., 201(2), 741-763. https://doi.org/10.1093/gji/ggv014

Schimmel, M., Stutzmann, E., and Ventosa, S. (2018). Low-frequency ambient noise autocorrelations: Waveforms and normal modes. Seismol. Res. Lett., 89(4), 1488-1496. https://doi.org/10.1785/0220180027

Schoenberg, M. (1980). Elastic wave behavior across linear slip interfaces. J. Acoust. Soc. Am., 68(5), 1516-1521. https://doi.org/10.1121/1.385077

Scuderi, M. M., Marone, C., Tinti, E., Di Stefano, G., and Collettini, C. (2016). Precursory changes in seismic velocity for the spectrum of earthquake failure modes. Nat. Geosci., 9(9), 695-700. https://doi.org/10.1038/ngeo2775

Sens-Schönfelder, C., and Wegler, U. (2006). Passive image interferometry and seasonal variations of seismic velocities at Merapi Volcano, Indonesia. Geophys. Res. Lett., 33(21), L21302. https://doi.org/10.1029/2006GL027797

Sens-Schönfelder, C., and Larose, E. (2010). Lunar noise correlation, imaging and monitoring. Earthq. Sci., 23(5), 519-530. https://doi.org/10.1007/s11589-010-0750-6

Sens-Schönfelder, C., Pomponi, E., and Peltier, A. (2014). Dynamics of Piton de la Fournaise volcano observed by passive image interferometry with multiple references. J. Volcan. Geoth. Res., 276, 32-45. https://doi.org/10.1016/j.jvolgeores.2014.02.012

Sens-Schönfelder, C., Snieder, R., and Li, X. (2019). A model for nonlinear elasticity in rocks based on friction of internal interfaces and contact aging. Geophys. J. Int., 216(1), 319-331. https://doi.org/10.1093/gji/ggy414

Shapiro, N. M., and Campillo, M. (2004). Emergence of broadband Rayleigh waves from correlations of the ambient seismic noise. Geophys. Res. Lett., 31(7), L07614. https://doi.org/10.1029/2004GL019491

Silver, P. G., Daley, T. M., Niu, F. L., and Majer, E. L. (2007). Active source monitoring of cross-well seismic travel time for stress-induced changes. Bull. Seismol. Soc. Am., 97(1B), 281-293. https://doi.org/10.1785/0120060120

Sleep, N. H. (2015). Shallow S-wave well logs as an indicator of past strong shaking from earthquakes on the Newport-Inglewood fault. Bull. Seismol. Soc. Am., 105(5), 2696-2703. https://doi.org/10.1785/0120150026

Snieder, R., Grêt, A., Douma, H., and Scales, J. (2002). Coda wave interferometry for estimating nonlinear behavior in seismic velocity. Science, 295(5563), 2253-2255. https://doi.org/10.1126/science.1070015

Takano, T., Nishimura, T., Nakahara, H., Ohta, Y., and Tanaka, S. (2014). Seismic velocity changes caused by the Earth tide: Ambient noise correlation analyses of small-array data. Geophys. Res. Lett., 41(17), 6131-6136. https://doi.org/10.1002/2014GL060690

Takano, T., Brenguier, F., Campillo, M., Peltier, A., and Nishimura, T. (2020). Noise-based passive ballistic wave seismic monitoring on an active volcano. Geophys. J. Int., 220(1), 501-507. https://doi.org/10.1093/gji/ggz466

Tanimoto, T., Eitzel, M., and Yano, T. (2008). The noise cross-correlation approach for Apollo 17 LSPE data: Diurnal change in seismic parameters in shallow lunar crust. J. Geophys. Res.: Planets, 113(E8), E08011. https://doi.org/10.1029/2007JE003016

Taylor, G., and Hillers, G. (2020). Estimating temporal changes in seismic velocity using a Markov chain Monte Carlo approach. Geophys. J. Int., 220(3), 1791-1803. https://doi.org/10.1093/gji/ggz535

Tsai, V. C. (2011). A model for seasonal changes in GPS positions and seismic wave speeds due to thermoelastic and hydrologic variations. J. Geophys. Res.: Solid Earth, 116(B4), B04404. https://doi.org/10.1029/2010JB008156

Wang, B. S., Yang, W., Wang, W. T., Yang, J., Li, X. B., and Ye, B. (2020). Diurnal and semidiurnal $P$ - and $S$-wave velocity changes measured using an airgun source. J. Geophys. Res.: Solid Earth, 125(1), e2019JB018218. https://doi.org/10.1029/2019JB018218

Wang, Q. Y., Brenguier, F., Campillo, M., Lecointre, A., Takeda, T., and Aoki, Y. (2017). Seasonal crustal seismic velocity changes throughout Japan. J. Geophys. Res.: Solid Earth, 122(10), 7987-8002. https://doi.org/10.1002/2017JB014307

Wang, Q. Y., Campillo, M., Brenguier, F., Lecointre, A., Takeda, T., and Hashima, A. (2019). Evidence of changes of seismic properties in the entire crust beneath Japan after the $M_{w}$ 9.0, 2011 Tohoku-oki Earthquake. J. Geophys. Res.: Solid Earth, 124(8), 8924-8941. https://doi.org/10.1029/2019JB017803

Weaver, R. L., Hadziioannou, C., Larose, E., and Campillo, M. (2011). On the precision of noise correlation interferometry. Geophys. J. Int., 185(3), 
1384-1392. https://doi.org/10.1111/j.1365-246X.2011.05015.x

Wegler, U., and Sens-Schönfelder, C. (2007). Fault zone monitoring with passive image interferometry. Geophys. J. Int., 168(3), 1029-1033. https://doi.org/10.1111/j.1365-246X.2006.03284.x

Xu, Z. J., and Song, X. D. (2009). Temporal changes of surface wave velocity associated with major Sumatra earthquakes from ambient noise correlation. Proc. Natl. Acad. Sci. USA, 106(34), 14207-14212. https://doi.org/10.1073/pnas.0901164106

Yamamura, K., Sano, O., Utada, H., Takei, Y., Nakao, S., and Fukao, Y. (2003). Long-term observation of in situ seismic velocity and attenuation. J. Geophys. Res., 108(B6), 2317. https://doi.org/10.1029/2002JB002005

Yang, W., Wang, B. S., Yuan, S. Y., and Ge, H. K. (2018). Temporal variation of seismic-wave velocity associated with groundwater level observed by a downhole airgun near the Xiaojiang fault zone. Seismol. Res. Lett., 89(3), 1014-1022. https://doi.org/10.1785/0220170282

Yao, H. J., and van der Hilst, R. D. (2009). Analysis of ambient noise energy distribution and phase velocity bias in ambient noise tomography, with application to SE Tibet. Geophys. J. Int., 179(2), 1113-1132. https://doi.org/10.1111/j.1365-246X.2009.04329.x

Zhan, Z. W., Tsai, V. C., and Clayton, R. W. (2013). Spurious velocity changes caused by temporal variations in ambient noise frequency content. Geophysi. J. Int., 194(3), 1574-1581. https://doi.org/10.1093/gji/ggt170

Zhang, Y. X., Planès, T., Larose, E., Obermann, A., Rospars, C., and Moreau, G. (2016). Diffuse ultrasound monitoring of stress and damage development on a 15-ton concrete beam. J. Acoust. Soc. Am., 139(4), 1691-1701. https://doi.org/10.1121/1.4945097

Zhao, P. P., Chen, J. H., Campillo, M., Liu, Q. Y., Li, Y., Li, S. C., Guo, B., Wang, J., and Qi S. H. (2012). Crustal velocity changes associated with the Wenchuan M8.0 earthquake by auto-correlation function analysis of seismic ambient noise. Chinese J. Geophys. (in Chinese), 55(1), 137-145. https://doi.org/10.6038/j.issn.0001-5733.2012.01.013 